This is the author's final, peer-reviewed manuscript as accepted for publication. The publisher-formatted version may be available through the publisher's web site or your institution's library.

\title{
Real-time detection of wave profile changes
}

Shing I Chang, Behnam Tavakkol, Shih-Hsiung Chou, Tzong-Ru Tsai

\section{How to cite this manuscript}

If you make reference to this version of the manuscript, use the following information:

Chang, S. I., Tavakkol, B., Chou, S. -H, \& Tsai, T. -R. (2014). Real-time detection of wave profile changes. Retrieved from http://krex.ksu.edu

\section{Published Version Information}

Citation: Chang, S. I., Tavakkol, B., Chou, S. -H, \& Tsai, T. -R. (2014). Real-time detection of wave profile changes. Computers \& Industrial Engineering, 75, 187-199.

Copyright: (C) 2014 Elsevier Ltd

Digital Object Identifier (DOI): doi:10.1016/j.cie.2014.05.020

Publisher's Link: http://www.sciencedirect.com/science/article/pii/S0360835214001739

This item was retrieved from the K-State Research Exchange (K-REx), the institutional repository of Kansas State University. K-REx is available at http://krex.ksu.edu 


\title{
REAL-TIME DETECTION OF WAVE PROFILE CHANGES
}

\author{
Shing I Chang ${ }^{1}$, Behnam Tavakkol ${ }^{2}$, Shih-Hsiung Chou ${ }^{1}$, Tzong-Ru Tsai ${ }^{3}$ \\ ${ }^{1}$ Quality Engineering Laboratory, Department of Industrial and Manufacturing Systems Engineering, \\ Kansas State University, USA \\ changs@ksu.edu, (785)532-3725 (Office), (785)532-3738 (Fax) \\ ${ }^{2}$ Department of Industrial and Systems Engineering, Rutgers University, USA \\ ${ }^{3}$ Department of Statistics, Tamkang University, Tamsui District, New Taipei City 25137 Taiwan
}

\begin{abstract}
S
A statistical process control (SPC) framework is proposed to detect potential changes of a wave profile on a real-time basis. In regular profile monitoring, change detection takes place when a complete profile is generated. In this study, the detection of a potential profile change takes place before the entire information on the profile of interest is fully available. The main research goal is to make a correct process decision as soon as possible. A real-world example of condensation-water-temperature profile monitoring was used to demonstrate the proposed framework. A simulation study was also conducted. The simulation results confirm that the proposed framework is capable of detecting profile changes without having to wait for the entire profile to be generated.
\end{abstract}

\section{Keywords: Exponentially Weighted Moving Average Filter, Hotelling $\mathrm{T}^{2}$ Chart, Statistical Process Control, Profile Analysis, Real-Time Monitoring}

\section{Introduction}

Profile monitoring has drawn much attention in the field of quality engineering in recent years. A profile is a relationship between a response variable and explanatory variable(s) (Woodall, 2007). Usually, the explanatory variable can be either time or space. If there are two spatial explanatory variables, the response is a surface. In this study, only one explanatory variable over time is considered for a response with oscillating patterns. In profile analysis, a decision about the quality of a profile is usually made at the end of the period when a profile is completely generated. Most of the research conducted in the field of profile monitoring is based on this approach. In this 
study, a new approach is proposed to detect profile changes based on real-time data feed before the entire profile is generated. The goal is to detect a possible deviation from a normal profile pattern as soon as possible.

It would be extremely beneficiary to detect an irregular profile before the entire profile is generated. In manufacturing, if profile changes related to a process status can be detected as soon as possible, product costs can be reduced through defect preventions. For example, Chang et al. (2012) studied the strategy of implementing SPC in a curing process in which the condensation-water-temperature profile is considered here. Figures 1 (a) to $(\mathrm{k})$ contain the progression of a water temperature profile during the curing process. In a separate work, the authors aimed to detect whether the curing process is in control or not based on the information provided in Figure 1(k) while this research focuses on monitoring the same process but using partial information provided by Figures 1 (a) to (j). If an abnormal profile be detected during earlier stages, process adjustments can be made to maintain product quality. The goal of this study is to provide a method to detect an out-of-control profile as soon and as accurate as possible. However, the practice of engineering process control (EPC) (del Castillo, 2002) is not in the scope of this study.

\section{Background}

\subsection{Current Profile Monitoring Methods}

Profile monitoring is the use of control charts for cases in which the quality of a process or product can be characterized by a functional relationship between a response variable and one or more explanatory variables (Woodall, 2007). In terms of modeling approaches for profile monitoring, Woodall (2007) specifies two categories of profile, linear and nonlinear profile. A review of the most conducted current research will be summarized in the following sub sections. The main purpose of this review is to show that none of current research in profile monitoring has considered wave profiles. In addition, none of the current research attempts to provide a decision before a profile is fully generated.

\section{Methods in Linear Profile Monitoring}

Methods dealing with linear profiles can be found in many studies. Kang and Albin (2000) proposed two methods to detect abnormal profiles. First, they monitored 
slope and intercept parameters using the Hotelling's $T^{2}$ control chart. Second, they used Exponential Weighted Moving Average (EWMA) and R chart to monitor average residuals between sample profiles and reference profile. Another model parameters monitoring methods can be found in Kim et al. (2003), Zou, et al. (2007), Mahmoud (2008), and, Zhu and Lin’s (2010) study. Kim et al. (2003) monitored slope, intercept, and the variance of deviation between samples and regression line simultaneously by their proposed three univariate exponentially weighted moving average (EWMA) charts. Zou, et al. (2007) proposed a multivariate exponentially weighted moving average monitoring scheme for linear profiles. Mahmoud (2008) monitored multiple linear regression model's parameters, intercept, slope, and variance, from the multiple linear profiles. Soleimani et al. (2009) dealt with simple linear profiles with a first-order autoregressive relationship among observations within a profile. Zhu and Lin (2010) proposed a Shewhart control chart for monitoring slopes of linear profiles from the truncated vertical density profiles problem (Walker and Wright, 2002).

Above mentioned methods dealing with linear profiles used the control charting approach. Mahmoud et al. (2006) proposed a change-point approach based on the segmented regression technique for testing the constancy of regression parameters in a linear profile data set. Hosseinifard et al. (2011) proposed a feed-forward neural network to detect and classify step shifts in linear profiles. More details regarding linear profiles monitoring methods can be found in Noorossana et al. (2011).

\section{Methods in Nonlinear Profile Monitoring}

To monitor the nonlinear profiles, Woodall (2007) categorized approaches into four types: (1) applying multiple and polynomial regression (Zou et al., 2007; Kazemzadeh et al. 2008; Mahmoud 2008); (2) applying nonlinear regression models (Ding et al., 2006; Williams et al., 2007; Shiau et al., 2009; Chang and Yadama 2010; Chen and Nembhard 2011); (3) use of mixed models (Jensen et al., 2008; Jensen and Birch, 2009; Qiu et al., 2010; Abdel-Salam, et al., 2013); and (4) use of wavelets (Reis and Saraiva, 2006; Zhou et al., 2006; Chicken et al., 2009). In this section, we will update recent developed methods according to these categories. Also, we will briefly introduce some represented approaches. Detail of other approaches to monitor the process stability can be found in Noorossana et al. (2011). 
Kazemzadeh et al. (2008) developed three methods for monitoring polynomial profiles in Phase I. These three methods are called the Change Point approach, FApproach and, the Hotelling $T^{2}$ Charting approach. They also developed a method based on the likelihood ratio test to identify the location of shifts.

Jensen et al. (2008) proposed a method of fitting the profiles for data where the within-profile measurements are correlated with each other, thus relaxing the assumption of independent errors. They did so by fitting a linear mixed model (LMM), which accounts for the correlation within profiles. The LMM also allows considering the profiles as a random sample of profiles from a common population distribution, which may be a more realistic assumption than assuming that the profiles are completely independent of each other.

Shiau et al. (2009) proposed a method for monitoring nonlinear profiles with random effects by nonparametric regression methods. They used the technique of principal components analysis to analyze the covariance structure of the profiles. Based on the principal components scores they proposed a monitoring scheme.

Chang and Yadama (2010) proposed a framework to monitor nonlinear profiles. Their framework was able to identify mean shifts or shape changes of profiles. They first applied Discrete Wavelet Transformation to remove noise from the profiles and then used B-splines to generate critical points to define the shape of profiles. Their methodology also allowed users to define number of segments that they would like to divide the profile into. A distance difference statistic for each segment provided diagnostic information. A Hotelling $T^{2}$ chart was used for multivariate control charting. As a further analysis for diagnosis, a decomposition method such as MTY decomposition (Mason et al., 1995) could be applied to the Hotelling $T^{2}$ statistics.

Abdel-Salam et al. (2013) proposed a semi-parametric mixed model approach to Phase I profile monitoring. Recently, in the absence of an obvious parametric model, nonparametric methods have been employed in the profile monitoring context. For situations where a parametric model is adequate over a part of the data but inadequate of other parts, the authors proposed a semi-parametric procedure that combines both parametric and nonparametric profile fits. They referred to their semi-parametric procedure as mixed model robust profile monitoring (MMRPM). For each approach of 
parametric, nonparametric and MMRPM methods, they proposed a Phase I Hotelling $T^{2}$ testing procedure to identify abnormal profiles based on the estimated random effects and obtain the corresponding control limits. Their simulation results showed that the MMRPM method performed well in making decisions regarding outlying profiles when compared to methods based on a miss-specified parametric model or based on a nonparametric regression method. They applied all three methods to the automobile engine data of Amiri et al. (2010), and found that the nonparametric method and the MMRPM method indicated signals that were not identified using parametric approaches only.

The literature on profile monitoring reviewed so far demonstrates that the wave profile has not been studied before. Furthermore, methods studied in this body of knowledge cannot be used to provide a solution before a profile is fully generated.

\subsection{Background of the curing process research}

The condensation-water-temperature profiles in a curing process (Chang, et al., 2012) are considered for process monitoring. This profile relates to one of the processes in producing high-pressure hose. This process is called curing. A curing process takes place in a sealed heat chamber, called an autoclave or vulcanizer (vulcanization 2010). High-pressure hose products in reels are loaded into the vulcanizer. Then the vulcanizer is heated according to a curing recipe to reach a set temperature for a fixed amount of time. The housing that contains a vulcanizer is often called vessel. Figure 2 shows a few condensation-water-temperature profiles collected during the curing process.

The traditional control charting approach on the condensation water temperature, $T_{t}$, does not work because $T_{t}$ is not stationary even though the process is in control. As explained in the previous section, the behavior of $T_{t}$ is dominated by the opening and closing of the water valve. Its mean level follows a functional (wave) form. In traditional control charting methods, this mean is usually a constant. But it is not the case here. A possible solution may be the use of moving center EWMA control chart (Montgomery, 2013). But this approach will not work either because the wave function is not limited to a fixed location. For example, at $\mathrm{t}=1$, the $\mathrm{T}_{1}$ observation can be large or small as long as the wave profile stays intact. As shown in Figure 2, there is no standard or gold template 
that can be used to describe the "normalcy" for $\mathrm{T}_{\mathrm{t}}$. For this reason, any attempt to try to fit the wave forms directly faces the same difficulty.

We have use of different types of functions such as the $2^{\text {nd }}$ order polynomial, $3^{\text {rd }}$ order polynomial, B-spline and, exponential decay to model the water-temperature profiles. Since these profiles look like waves, they are also called the wave profiles. We also consider the use of fast Fourier transform to analyze these wave forms. It turns out that the transformed signals do not simply the original wave forms because a typical wave form in this case does not have a constant frequency or amplitude. Finally, the sum of areas generated from the enclosure between the exponential-decay cutting line and the wave profile can be used as a measure for further implementations. The main reason for adopting the sum of areas statistic is that this statistic is about an overall estimate of how the general wave pattern of a cycle is generated rather than an attempt to find the exact location of each detailed peak and valley. The detail of the proposed method is introduced in the next section.

\section{Proposed Methods}

Our proposed strategy is to convert each wave profile using exponential-decayed function as cutting line into a statistic that can be directly fed into a univariate control chart that is available in any SPC software.

During the phase I SPC, assume $m$ in-control wave profiles are collected and each contains $n$ observations. The following exponential-decayed function is defined as:

$$
Y_{i j}=\exp \left(a_{i} X_{i}^{b_{i}}\right), i=1,2, \ldots, m, j=1,2, \ldots, n,
$$

where $Y_{i j}$ and $X_{i}$ are the $i^{\text {th }}$ wave profile and observed time for the $j^{\text {th }}$ sample; $a_{i}, b_{i}$ are unknown parameters to be estimated using least square calculated based on given incontrol wave profiles.

The exponential-decayed cutting line cuts through a wave profile and forms areas as shown in Figure 3. The sum of areas from $m$ in-control samples of wave profiles was denoted as $X_{i}^{\prime}$. A polynomial model in equation (3) was proposed to account for the number of peaks and valleys that were different from cycle to cycle. 


$$
\log \left(X_{i}^{\prime}\right)=\beta_{0}+\beta_{1} z_{i}+\beta_{2} z_{i}^{2}+\epsilon_{i}, i=1,2, \ldots, m
$$

where $z_{i}$ is the number of waves of the $i^{\text {th }}$ profile. For example, in Figure 3 , there are 28 waves in the profile.

A second-order polynomial model was then fit to remove noise due to the number of waves in each profile. Standardized residuals of this regression model form the quality characteristic for control charting:

$$
e_{i}=\log \left(X_{i}^{\prime}\right)-\left(\hat{\beta}_{0}+\hat{\beta}_{1} z_{i}+\hat{\beta}_{2} z_{i}^{2}\right)
$$

The standardized residuals should be independent and normally distributed. If the independent assumption was not met, an exponentially weighted moving average (EWMA) filter could be applied to remove the autocorrelation. The obtained statistic was then used for control charting purposes. Any control chart for individual observations such as the individual-X (IX) or EWMA control chart was used as an appropriate tool to monitor the residuals. Note that IX chart is very sensitive to normal assumption. Therefore, one should check the normality assumption of the standardized residuals in equation (3) before an IX chart is used.

We will use the same data set of the wave profiles for this research. Once again, the research goal is to detect a profile change as fast as possible before a curing process ends. It means that we would like to identify an abnormal wave pattern during, not after, a curing cycle.

Figure 4 depicts the relationship of the original process monitoring approach based on entire profiles and the proposed research based on a partial profile. Figure 4(a) is a control chart for monitoring the curing process using complete profiles. The one point that plots outside the control limit indicates the present profile is abnormal and implies that the process is out of control. Figure 4(b) demonstrates that the proposed research uses a pair of control charts to detect this out-of-control process when only a quarter of information of this profile is obtained. In the following section, we will outline a couple of potential solutions to achieve the task illustrated in Figure 4(b).

Note that the proposed pair of IX and Moving Range (MR) control charts in Figure 4(b) are different from the traditional implementation of control charts in three aspects. First, each data point in the proposed charts is correlated with points preceeding 
it. This is due to the fact that an exponential curve is fit to the partial profile available up to that point. Second, the scope of the proposed control charts are limited to a fraction as oppose to the usual sample number, for example, 0.5 meaning $50 \%$ of the entire profile. Once the entire profile is generated, no new data point is to be plotted on the control charts. Finally, the proposed control charts do not rely on the same assumption of traditional control charting where an assigable cause may last from batch to batch until the cause is removed. In other words, an assignable cause may only exist in the current batch or production cycle only. We only rely on the information at a current batch for decision making. If an assignable cause lasts from batch to batch, the proposed method will still work.

The proposed methodologies in this research were motivated by the curing process demonstrated earlier. The curing process takes a fixed time unit for which we assign a number $100 \%$ cured or simply 1 without loss of generality. We divided the typical time frame of curing in this study into eleven portions represented by the fractions: $1 / 8,1 / 7,1 / 6,1 / 5,1 / 4,1 / 3,1 / 2,2 / 3,3 / 4,4 / 5$, and 1 . For example, $1 / 2$ represents that a half of the wave profile or the data from the beginning to the midway point is obtained so far. Prior knowledge is needed to set up appropriate number of portions because it depends on the pattern and location of the changes in abnormal profiles which distinguish them from the normal profiles. In this case, eleven portions were enough for the analyses. In the next step, 100 normal profiles were selected for Phase I analyses. An exponential-decay function was used to model these 100 profiles. The sums of the areas generated from the enclosure between the exponential-decay cutting lines and the wave profiles were calculated for them. This calculation was done 11 times due to 11 different portions of the wave profile mentioned earlier. We now consider two possible phase II approaches for process monitoring.

\subsection{Method I (Filtering then Standardizing)}

The first proposed methodology is displayed in Figure 5. We consider variable $X_{i t}$ as the accumulated area for the profile $i$ from the starting point up to the point $t$. The time index $t$ can gain different values but in the special case of curing process we only define eleven values of $1 / 8,1 / 7,1 / 6,1 / 5,1 / 4,1 / 3,1 / 2,2 / 3,3 / 4,4 / 5$, and 1 as the time index. Note that this choice does not produce equal time intervals for the entire profile. One 
advantage is that it provides more frequent check points at the beginning of the profile generation. This practice will lead to more opportunities for detections. It is clear that for profile $i$, different values of $X_{i t}$ at different times are dependent and correlated because the cumulated areas at a later stage contain those in earlier stages. We plotted both the autocorrelations (ACF) and partial autocorrelations (PACF) to confirm that this is true. In order to remove the correlation from $X_{i t}$ we needed to apply a filter as in Figure 5 .

According to the ACF and PACF we could conclude that the best type of filter to remove the autocorrelation in this case would be an AR(1) filter. This was because of having a single spike at the beginning of the PACF graph. After applying the AR(1) filter, the uncorrelated variable $X_{i t}$ was named $X^{\prime}{ }_{i t}$. Note that $X^{\prime}{ }_{i t}=X_{i t}-\hat{X}_{i t}$ where $\hat{X}_{i t}$ is the estimated value of the regression between $X_{i t}$ and $X_{i(t-1)}$. This process works only because the underlying autocorrelation is $\mathrm{AR}(1)$.

Based on this uncorrelated data series from all profiles in Phase I, we then calculated the appropriate mean cumulated area $\mu_{t}$ and its standard deviation $\sigma_{t}$ at time $t$. We assume that all profiles used in phase I are representative of an exemplary process. Finally the uncorrelated series $X^{\prime}{ }_{i t}$ is standardized to generate a standardized series $e_{i t}$ (shown in equation (4)) which is then ready to be fed into any control chart for individual observations.

$$
e_{i t}=\frac{X_{i t}^{\prime}-\mu_{t}}{\sigma_{t}}
$$

\subsection{Method II (Segmenting then Standardizing)}

Since the overlapping areas cause autocorrelation, this second proposed method aims to avoid the autocorrelation by redefining the accumulated areas. This method is depicted in Figure $\underline{6}$. $D_{i t}$ was defined as the difference of the enclosed areas for profile $i$ between time $t-1$ and time $t$. Thus $D_{i t}$ was calculated according to the following formula in equation (5).

$$
D_{i t}=X_{i t}-X_{i(t-1)}
$$


Note that $D_{i t}$ is in fact the accumulated areas between $t-1$ and $t$. Since the non-overlap areas are taken into account, statistics, $D_{i t}$, are independent. We now standardize $D_{i t}$ according to equation (6):

$$
D_{i t}^{\prime}=\frac{D_{i t}-\mu_{t}^{\prime}}{\sigma_{t}^{\prime}}, \quad t=1 / 8,1 / 7, \ldots, 1,
$$

where $\mu_{t}^{\prime}$ is the average of $D_{i t}$ 's of 100 Phase I profiles at time $t$, and $\sigma_{t}^{\prime}$ is the standard deviation of $D_{i t}$ 's of 100 Phase I profiles at time $t$. The standardized statistics, $D_{i t}^{\prime}$ is then used for further analysis. The major advantage of using this approach over the first method is that $D_{i t}^{\prime}$ 's are not autocorrelated. Hence, there is no need to apply any type of filtering to remove the autocorrelation. Note that $D_{i t}^{\prime}$ are i.i.d. standard normal variables at $t=1 / 8,1 / 7, \ldots, 1$. During the phase II monitoring, any sample $i$ will generate a $D_{i t}^{\prime}$ value to be plotted on a pair of standardized control charts as shown in the next section.

\subsection{Control Charting}

We can now plot either $e_{i t}$ (method I: eq. (4)) or $D_{i t}^{\prime}$ (method II: eq. (6)) on a pair of IX and MR control charts. Since both statistics have been standardized, the control limits for the IX and MR control charts are defined in equations (7) and (8), respectively. Having $n=2$, values of $d_{2}, D_{3}$ and $D_{4}$ are set as 1.128, 0 and 3.267 respectively. For details, see Montgomery (2013).

IX chart:

$$
U C L=\bar{X}+3 \frac{\overline{M R}}{d_{2}}=3 \frac{\overline{M R}}{1.128}=3
$$

$$
\begin{aligned}
& C L=\bar{X}=0 \\
& L C L=\bar{X}-3 \frac{\overline{M R}}{d_{2}}=-3 \frac{\overline{M R}}{1.128}=-3
\end{aligned}
$$

MR chart:

$$
\begin{aligned}
& U C L=D_{4} \overline{M R}=3.267 \overline{M R}=3.685 \\
& C L=\overline{M R}=1.128 \\
& L C L=D_{3} \overline{M R}=0
\end{aligned}
$$

Depending on the proposed method used, the MR for profile $i$ is defined as either $M R_{i t}=\left|e_{i t}-e_{(i-1) t}\right|$ or $M R_{i t}=\left|D_{i t}^{\prime}-D_{(i-1) t}^{\prime}\right|$ at any location $t=1 / 8,1 / 7, \ldots, 1$. In 
many applications, the MR of two successive observations is used as the basis of estimating the process variability.

During the Phase I of control charting, a sample of representative observations should be collected to calculate $\bar{X}$ and $\overline{M R}$. Normality assumption is important when IX chart is used. We should point out the both the EWMA and CUSUM (Cumulative Sum) control charts are the other competent control charts to replace the IX chart for individual observations. The normality assumption is less of an issue for the EWMA and CUSUM charts. Although there are EWMA and CUSUM based control charts for monitoring process variations, the MR chart is a much simpler method if the normality assumption is not an issue. For more details, please refer to Montgomery (2013).

Figure 7 demonstrates when IX and MR charts are used to detect potential out-ofcontrol profiles. The mean and standard deviation of $D_{t}$ (see equation (4)) based 100 normal profiles were calculated and summarized in Table 1. These values were used for equations (6), (7), and (8) for control charting. As illustrated in Figure 4(a), the proposed method was able to detect that that profile was abnormal right at $1 / 3$ point of the profile generation before the entire profile was generated. Yet, in order to validate the performance of the second method, further analysis is needed. A simulation study will be conducted based on multiple runs in addition to the real-world profiles from a single case.

\section{A Simulation Study}

Two types of profiles are simulated. First type of profiles, are those that are deemed normal profiles for an in-control process. These normal profiles are the ones that have a descending shape. An exponential decay function is chosen as the best fit to them. An example of real-world profiles of this type is shown in Figure $\underline{9}$ (a) while a simulated profile is shown in Figure 9 (b). Second type of profiles, are called abnormal profiles, those have inadequate frequency, i.e., number of peaks and valleys, and large amplitudes. There are six scenarios simulated to be abnormal profiles as shown in Figure 10. Note that a solid line represents a simulated normal profile while a dash line represents simulated abnormal profile. In scenario 1, the number of peaks and valleys is significantly small. In addition, the amplitudes after 300 time units is small as well. 
Scenario 2 shows that the wave patterns are acceptable before 350 time unit but become flat after 350. In scenario 3, not only the number of peaks and valleys is small but the profile patterns shift downward and become flat after 200 time unit. Scenarios 4 to 6 are all large amplitudes with different frequencies.

\subsection{Design of Experiments}

The purpose of the simulation study is to evaluate the performance of the proposed method. Both phase I and phase II samples were generated. We first generated 500 normal profiles to establish the proposed IX and MR charts then we generated another set of 200 random mixtures of 100 normal and 100 abnormal profiles for validation. First 500 normal profiles shape as shown in Figure 9 (b) were generated and labeled as $y(t)$ from a descending shape and exponential-decay function defined below:

$$
\begin{aligned}
& x(t)=e^{-n t}\left(f \times \cos (m t)+C(1-\cos (m t))-\frac{C\left(\frac{t}{T}-\sin (m t)\right)}{m T}\right) \\
& \text { and } \\
& y(t)=f+2 A \frac{\left(x(t)-\frac{\max (x(t))+\min (x(t))}{2}\right)}{\frac{\max (x(t))-\min (x(t))}{2}}, \quad t=0, \ldots, T,
\end{aligned}
$$

where $x(\mathrm{t})$ is the simulated water temperature at time $t, y(t)$ is the scaled water temperature at time $t, f$ is the scaling parameter of the water temperature, $A$ is the amplitude parameter. $e^{-n t}$ is the damping factor where $n$ determines speed of the damping, $m$ is the controller parameter of frequency of the wave, $t$ accounts for time, and $T$ is the time at the end of the profile. Intervals of 0.05 were used for $t$ in this model. The default $T$ in this study is 24.95 , so that the number of data points within a profile will be 500. Each parameter in equations (9) and (10) is simulated as a normally distributed random variable. To resemble real-world, condensation-water-temperature profiles, we chose to use the following settings: $\mathrm{A} \sim N\left(10,0.7^{2}\right), f \sim N\left(260,2^{2}\right), n \sim N\left(0.1,0.005^{2}\right)$, and $m \sim N\left(5,0.1^{2}\right)$ where the first parameter in the normal distribution is the mean and the second parameter is the standard deviation . 
In order to simulate abnormal profiles for each scenario, equation (9) and (10) as well as equation (11) and (12) are obtained. Note that, the parameters $f, m$, and $A$ in the equation (11) and (12) are all followed by normal distribution, as well. All parameters setting for generating abnormal profiles are shown in Table 2.

$$
\begin{gathered}
x(t)=f \cos (m t) \\
y(t)=f+2 A \frac{\left(x(t)-\frac{\max (x(t))+\min (x(t))}{2}\right)}{\frac{\max (x(t))-\min (x(t))}{2}}, t=0, \ldots, T .
\end{gathered}
$$

The statistics of the average time of the proposed methods to alarm for out of control is studied via the following procedure:

Step 1: The time domain, $T$, is set as 24.95 for every profile so that all of the profiles consisted of 500 observed points.

Step 2: Generate 500 in-control profiles as the Phase I sample based on functions (9) and (10). Calculate control chart parameters using the monitoring procedure in Section 3. See equations (7) and (8).

Step 3: Generate a Phase II sample for each scenario, in which 100 in-control profiles generated from equations (9) and (10) are randomly mixed with 100 out-ofcontrol profiles generated from settings in Table 2.

Step 4: Use the monitoring parameters calculated in Step 2 to detect the out-of-control signal from the Phase II sample generated in Step 3. Control chart performance statistics are collected.

Step 5: Repeat Step 2 to Step 45000 times. Statistics of mean and standard deviation of first detection time are generated.

\subsection{Simulation outcomes}

Based on the simulation procedure listed in the previous section, out of 200 phase II profiles generated 100 were normal profiles and 100 were abnormal profiles with various scenarios. Table 3 summarizes the simulation results of proposed methods among all scenarios. In Table 3, the true positives (TP) are the number of normal profiles 
assigned to the normal group, while the true negatives (TN) are abnormal profiles classified as the abnormal group. If normal profiles are assigned to the abnormal group, they are called false negatives (FN) known as type I error. On the other hand, when abnormal profiles are classified to the normal group, they are called false positives (FP) as known type II error. Note that average run length (ARL) is not used to compare both methods because the "run length" in this study is really how fast to detect a shiftedprofile before the entire profile is generated. But in the case when the entire profile is used for monitoring, the traditional "run length" is the number of profiles generated before the control chart detects an out-of-control point. Note that there are only 11 monitoring spots in the wave profile in this case.

The accuracy rate in Table 3 defined in equation (14) is a more meaningful statistic than ARL. The accuracy is a function of sensitivity and specificity defined in equations (15) and (16), respectively. Sensitivity and specificity are known as the proportion of positive tuples and negative tuples are all correctly identified. The accuracy rate is a good indicator for comparison because it provides overall performance criteria in one value - the higher the better. For more details of accuracy rate, please refer to Han et al. (2006).

In this simulation study, the average type I error of method I and method II among all scenarios is 0.02 and 0.01 , respectively. On the other hand, type II error of both methods is all 0 , meaning that the proposed method is capable of detecting all abnormal profiles. With regards to accuracy rate of method I and method II, both methods provide high accuracy rate, i.e., 0.99 and 0.995 . The next question is then: "How fast the proposed methods can detect abnormal profiles?”

$$
\begin{gathered}
\text { accuracy = sensitivity } \frac{(T P+F N)}{(T P+F N+F P+T N)}+\text { specificity } \frac{(F P+T N)}{(T P+F N+F P+T N)} \\
\text { sensitivity }=\frac{T P}{(T P+F N)} \\
\text { specificity }=\frac{T N}{(F P+T N)}
\end{gathered}
$$

According to Table 3 , the mean of average detection time of method I and method II among all scenarios is 0.14 and 0.16 , respectively. In other words, both methods can 
detect an abnormal profile between 1/8 and 1/6 of the entire profile before the end of the process. Figure 11 provides more details of the average detection time of method I and method II in all scenarios. Since both methods' standard deviations of detection time are all small, we only compare their average detection time. From Figure 11, average detection times from both methods show that method I is faster than method II in in scenarios 1, 2, and 3, while there is no difference between method I and method II in scenarios 4, 5, and 6. Taking scenario 2 for an example, we found that abnormal profiles in scenario 2 of method I show better detecting power in term of the average detection time than method II. In other words, method I can detect abnormal profiles faster than method II.

In summary, although the accuracy rate of both methods is very close, method I detects abnormal profiles in scenarios 1, 2, and 3 faster than method II does. Also, in scenarios 4,5 , and 6 , there is no difference between method I and method II in term of average detecting time. We recommend method I for its high accuracy rate and faster detection time. However, we can also recommend method 2 although it is less efficient but it detect in advance the profile behavior.

\section{A Case Study}

In this case study, out of the 186 wave profiles of the curing process obtained from March to April of 2011, and 91 of them were selected to construct the phase I process because their shapes are similar to the typical waveform profiles. We also pick three abnormal waveform profiles to test the robustness of the proposed methods. Figure 12 shows three abnormal profiles (labeled abn1 to abn3) superimposed on 91 typical waveform profiles (labeled typical) that will be examined in this study. Abn1 indicates the valve was normal before time 400, while abn2 shows the thermocouple stopped working around time 100. Abn3 gives information that the valve was not closed for a period of time between time 10 and 100 , as well as time 110 to 410 .

The objective of applying method I and method II to those three abnormal profiles is to investigate if the proposed methods can detect the abnormal profiles before the entire process is finished. The results of method I are shown in Figure 13. Figure 13 (a) shows the abn1 stayed in control before the 8th observation. In other words, before 2/3 of the 
manufacturing, the process was normal. For abn2 and abn3 on the Figure 13 (b) and (c), both results show the method I catch the abnormal right after $1 / 7$ of the process.

The results of method II are shown in Figure 14. In Figure 14 (a), the method II indicates the out-of-control signal occurred during 1/7 of the process for abn1. And Figure 14 (b) and (c) state the out-of-control happened in 1/5 and 1/4 of the process, respectively. Note that control limits and center line are drawn very close together in Figures 13 (b) and 14 (b) because the out-of-control points are very large.

Both results described above show that method I and method II are capable of detecting the abnormal waveform profiles. For abn1, method I signals out-of-control after 2/3 of the process, but method II states there is out-of-control starting from 1/7 of the process. Although method II detect abnormal signal faster than the method I does, the out-ofcontrol signals that provided by method II for abn1 are actually false alarm. In Figure 12, the abn 1 profile is classified normal profile between time 0 to time 400 , however, the method II indicates out-of-control signal starting from time 71. Therefore, for abn1, the method I provides better result than method II does. For abn2 and 3, method II detects out-of-control signals slower than method I does. Therefore, method I is our recommended approach to detect real-time waveform profiles monitoring.

\section{Conclusions}

In this research, the process monitoring task for wave profiles is based on realtime detection before entire profile information is obtained. The goal is to detect an abnormal profile at an earlier stage of profile generations. A curing process has been studied to demonstrate the proposed concept.

We have studied two potential solutions but favored the first approach because of its high accuracy rate and fast detection time. The proposed method includes the following steps: determining the desired decision times ( $t$ 's), modeling the exponential decay function to the set of profiles at time $t$, calculating the values of enclosed areas of the profile over time, calculating the $\mathrm{AR}(1)$ filter of the enclosed areas and subtracted to the observation for each profile, and standardizing the statistics for each profile using the mean and standard deviation of the statistics obtained from the Phase I profiles. Finally, 
the statistics obtained from these procedures for each of the profiles are fed to IX and MR control charts for process monitoring.

Simulations provide various controlled out-of-control scenarios mixed with normal profiles. Six types of abnormal profiles in the simulation study included different numbers of waves and flat line starting at middle or at the end of process. Simulation results demonstrated that the proposed methods are capable of detecting these abnormal profiles although some false alarms were also generated. And both methods provide high accuracy rate. Furthermore, the proposed methods caught these abnormal profiles before an entire profile needed to be generated, i.e., between 1/8 and 1/6 of entire profile.

The proposed method can be extended to other applications in which the quality characteristic of interest exhibits an oscillating pattern. For example, Hammond et al. (2013) consider a flow pattern from a syringe pump of an automated instrument for veterinary diagnostic. When extreme amplitudes emerge in the wave pattern, a failure mode occurs and results in yield loss and rework.

Further research is needed to diagnose a process at any portion of a profile. The proposed method only calculated means and standard deviations at 11 fixed locations. There may be times that a diagnosis is required not necessarily at these locations. Monitoring a process at each data-collection time unit demands huge amounts of computations and is not feasible. A multivariate analysis approach can also be utilized for change detections with real-time data feed. The challenge for a multivariate approach is its huge amount of computations for estimating the variance covariance matrices at each profile segment. Further research is needed to overcome this computational obstacle and enhance its real-time detection functionality. 


\section{Acknowledgments}

We would like to thank the editor and several reviewers for insightful suggestions which make the presentation of this paper better. The authors would also like to thank the grant from National Science Council, Republic of China, provided to Professor Tzong-Ru Tsai who visited the IMSE Department at Kansas State University during 2011-2012 academic year. Parts of this research are based Mr. Behnam Tavakkol's M.S. thesis at Kansas State University. 


\section{Bibliography}

Abdel-Salam, A.S.G., Birch, J.B., and Jensen, W.A. (2013). A Semiparqametric Mixed Model Approach to Phase I Profile Monitoring. Quality and Reliability Engineering International, 29(4), 555-569.

Amiri, A., Jensen, W.A., and Baradaran Kazemzadeh, R. (2010). A Case Study on Monitoring Polynomial Profiles in the Automative Industry. Quality and Reliability Engineering International , 26(5), 509-520.

Chang, S. I., and Yadama, S. (2010). Statistical Process Control for Monitoring Non-linear Profiles Using Wavelet Filtering and B-Spline Approximation. International Journal of Production Research, 48(4), 1049-1068.

Chang, S., Tsai, T., Lin, D., Chou, S.H., and Lin, Y. (2012). Statistical Process Control for Monitoring Nonlinear Profiles: a Six Sigma Project on Curing Process. Quality Engineering, 24(2), 251-263.

del Castillo, E. (2002). Statistical Process Adjustment for Quality Control. NY: Wiley.

Chen, S. and Nembhard, H.B., (2011). A High-dimensional Control Chart for Profile Monitoring, Quality and Reliability Engineering International, 27(4), 451-464.

Chicken, E. Pignatiello, Jr., J., andSimpson, J.R. (2009) Statistical Process Monitoring of Nonlinear Profiles Using Wavelets, Journal of Quality Technology, 41(2), 198-212.

Ding, Y., Zeng, L., and Zhou, S. (2006). Phase I Analysis for Monitoring Nonlinear Profiles in Manufacturing Processes, Journal of Quality Technology, 38(3), 199-216.

Hammond, J., Haiss, A., Lavigne, S., Daniels, B., and Allen, J. (2013). A Functional Approach to Fixing Flow Oscillation. Quality Engineering, 25(4), 385-391.

Han, J., and Kamber, M. (2006). Data mining concepts and techniques (2nd ed.). Amsterdam: Elsevier

Hosseinifard, S., Abdollahian, M., and Zeephongsekul, P. (2011). Application of Artificial Neural Network in Linear Profile Monitoring. Expert Systems with Applications, 38(5), 49204928.

Jensen, W.A. and Birch, J.B. (2009). Profile Monitoring Via Nonlinear Mixed Models, Journal of Quality Technology 41(1), 18-34.

Jensen, W.A., Birch, J.B., and Woodall, W.H. (2008). Monitoring Correlation within Linear Profiles using Mixed Models, Journal of Quality Technology, 40(2), 167-183.

Kang, L.; Albin, S. L. (2000). On-Line Monitoring when the Process Yields a Linear Profile, Journal of Quality Technology, 32(4), 418-426. 
Kazemzadeh, R., Noorossana, R., and Amiri, A. (2008). Phase I Monitoring of Polynomial Profiles. Communications in Statistics--Theory and Methods, 37(10), 1671-1686.

Kim, K., Mahmoud, M. A., and Woodall, W. H., (2003). On the Monitoring of Linear Profiles. Journal of Quality Technology, 35(3), 317-328.

Mahmoud, M. A. (2008). Phase I Analysis of Multiple Regression Linear Profiles, Communications in Statistics - Simulation and Computation, 37(10), 2106-2130.

Mahmoud, M. A., Parker, P. A., Woodall, W. H., and Hawkins, D. M. (2006). A Change Point Method for Linear Profile Data. Quality and Reliability Engineering International, 247268.

Mason, R., Tracy, N., and Young, J. (1995). Decomposition of T2 for Multivariate Control Chart Intepretation. Journal of Quality Technology, 27(2), 109-119.

Montgomery, D. C. (2013). Introduction to Statistical Quality Control (7th ed.). Wiley.

Noorossana, R. S. (2011). Statstical Analysis of Profile Monitoring. Hoboken, New Jersey: John Wiley \& Sons, Inc.

Qiu, P., Zou, C. and Wang, Z. (2010). Nonparametric Profile Monitoring by Mixed Effects Modeling, Technometrics, Vol. 52(3):265-277.

Reis, M.S., SARAIVA, P.M. (2006). Multiscale Statistical Process Control of Paper Surface Profiles, Quality Technology and Quantitative Management, 3(3), 263-282.

Shiau, J.J.H., Huang, H.L., Lin, S.H., and Tsai, M.Y. (2009). Monitoring Nonlinear Profiles with Random Effects by Nonparametric Regression, Communications in Statistics-Theory and Methods, 38(10), 1664-1679.

Soleimani, P., Noorossana, R., and Amiri, A. (2009). Simple Linear Profiles Monitoring in the Presence of within Profile Autocorrelation, Computers \& Industrial Engineering, 57, 3, 10151021.

Vulcanization. (2010). In Encyclopaedia Britannica. Retrieved September 10, 2010, from Encyclopædia Britannica Online: http://www.britannica.com/EBchecked/topic/633433/vulcanization

Walker, E. and Wright, S. P. (2002). Comparing Curves Using Additive Models, Journal of Quality Technology, 34(1), 118-129.

Williams, J. D., Woodall, W. H., and Birch, J. B. (2007). Statistical Monitoring of Nonlinear Product and Process Quality Profiles, Quality \& Reliability Engineering International, 23(7), 925-941. 
Woodall, W.H. (2007). Current Research on Profile monitoring, Produção 17(3): 420-425.

Zhou, S.Y., Sun, B.C., and Shi, J.J. (2006). An SPC Monitoring System for Cycle-based Waveform Signals using Haar Transform. IEEE Transactions on Automation Science and Engineering, $3(1), 60-72$.

Zhu, J., and Lin, D. K. (2010). Monitoring the Slopes of Linear Profiles. Quality Engineering, 22(1), 1-12.

Zou, C.,Tsung, F., and Wang, Z. (2007). Monitoring General Linear Profiles Using Multivariate Exponentially Weighted Moving Average Schemes, Technometrics, 49(4), 395-408. 


\section{Figures}

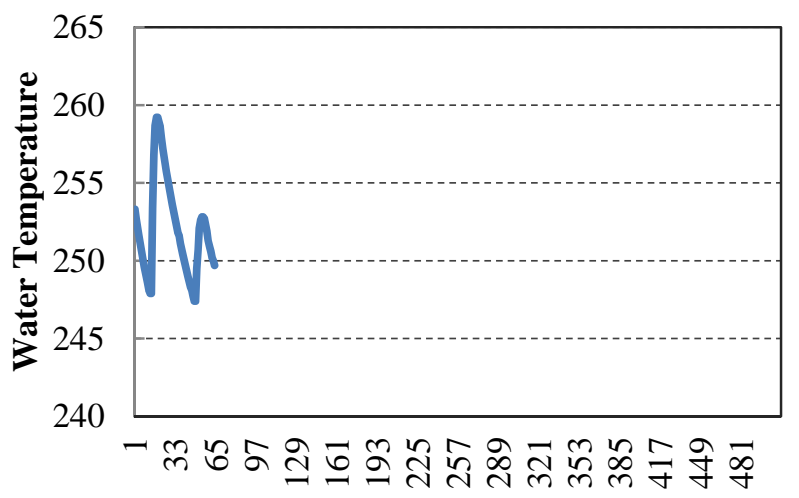

Time

(a) $1 / 8$ of profile

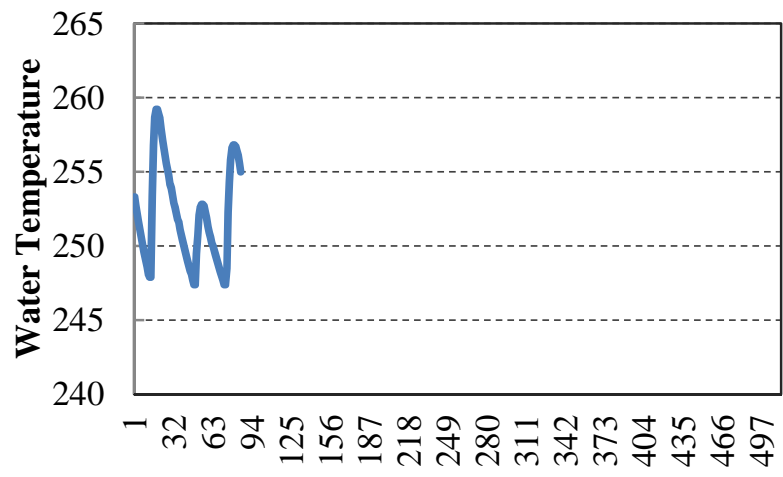

Time

(c) $1 / 6$ of profile

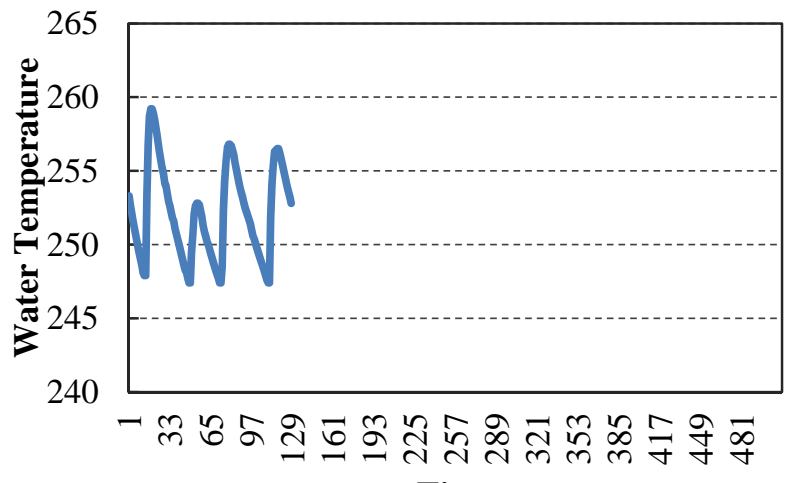

Time

(e) 1/4 of profile

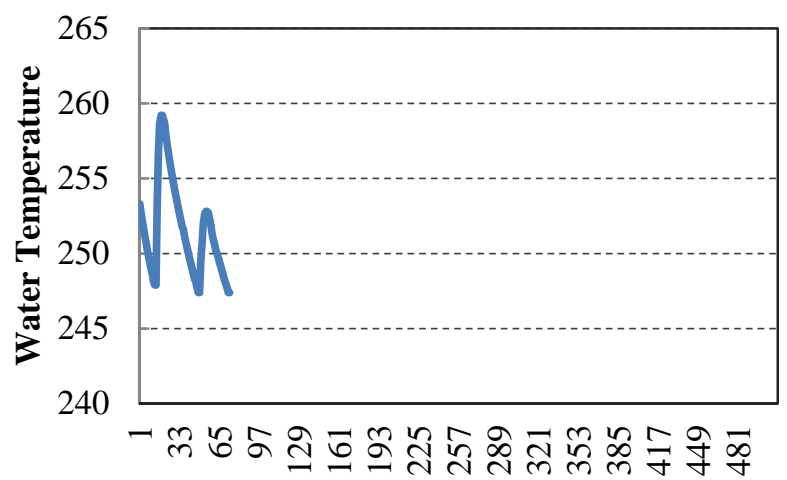

Time

(b) $1 / 7$ of profile

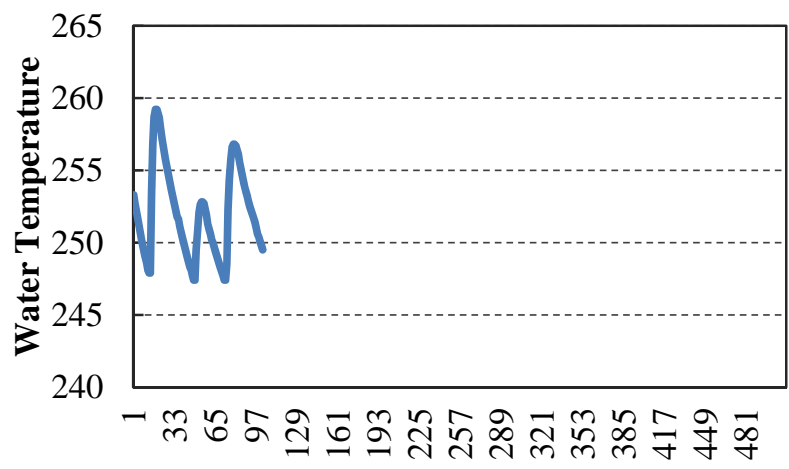

Time

(d) 1/5 of profile

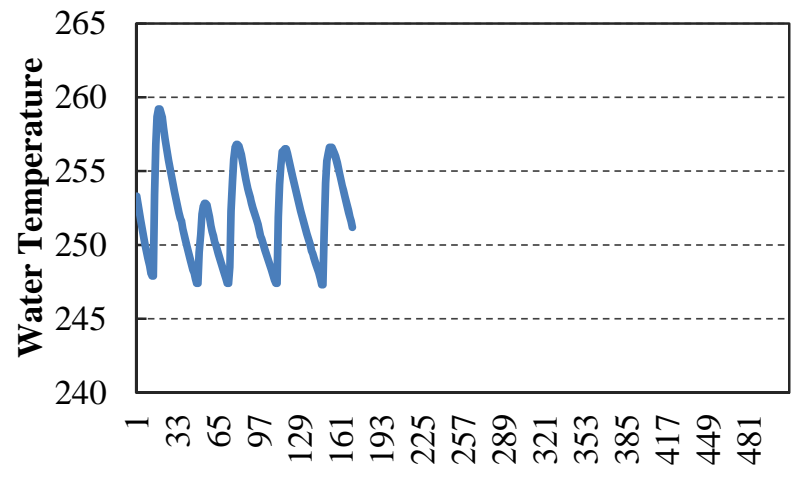

Time

(f) $1 / 3$ of profile 


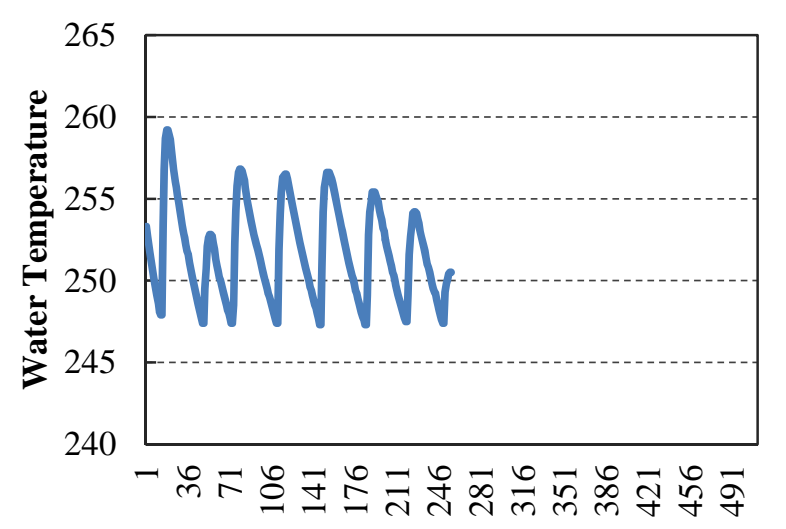

Time

(g) $1 / 2$ of profile

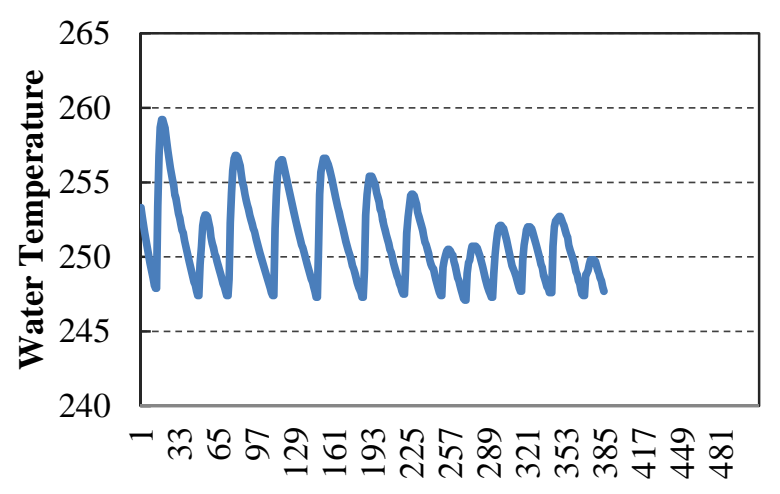

Time

(i) 3/4 of profile

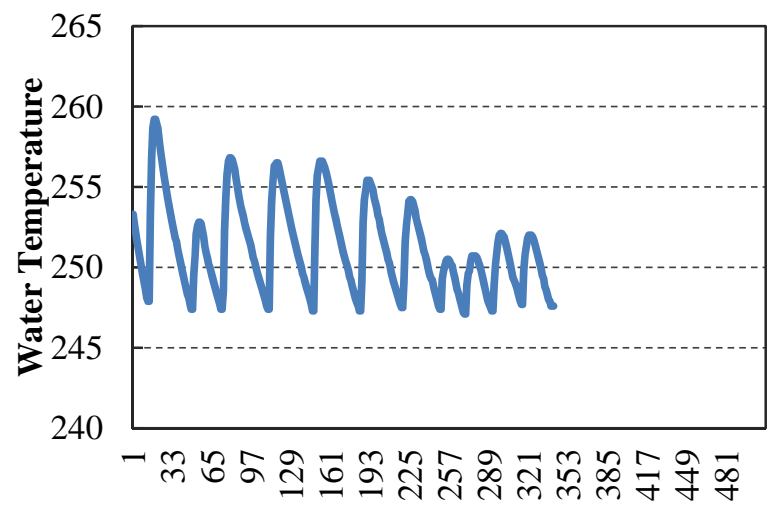

Time

(h) $2 / 3$ of profile

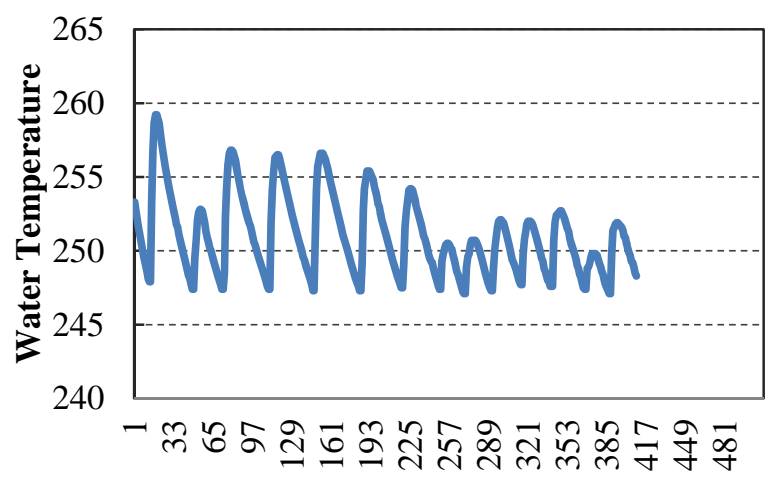

Time

(j) $4 / 5$ of profile

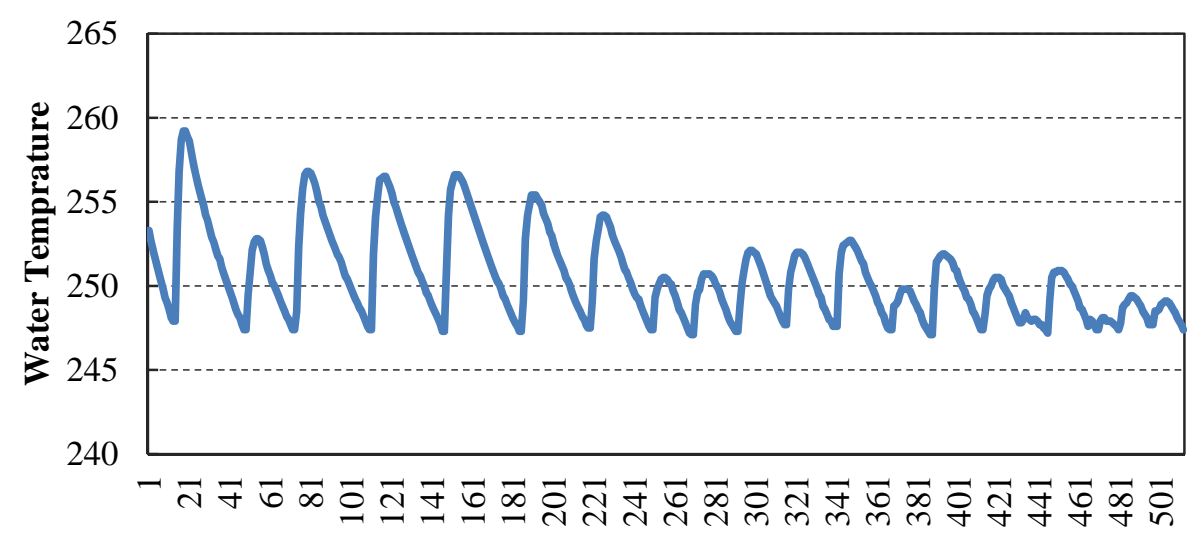

Time

(k) Complete profile

Figure 1. Completion of a condensation-water-temperature profile. 


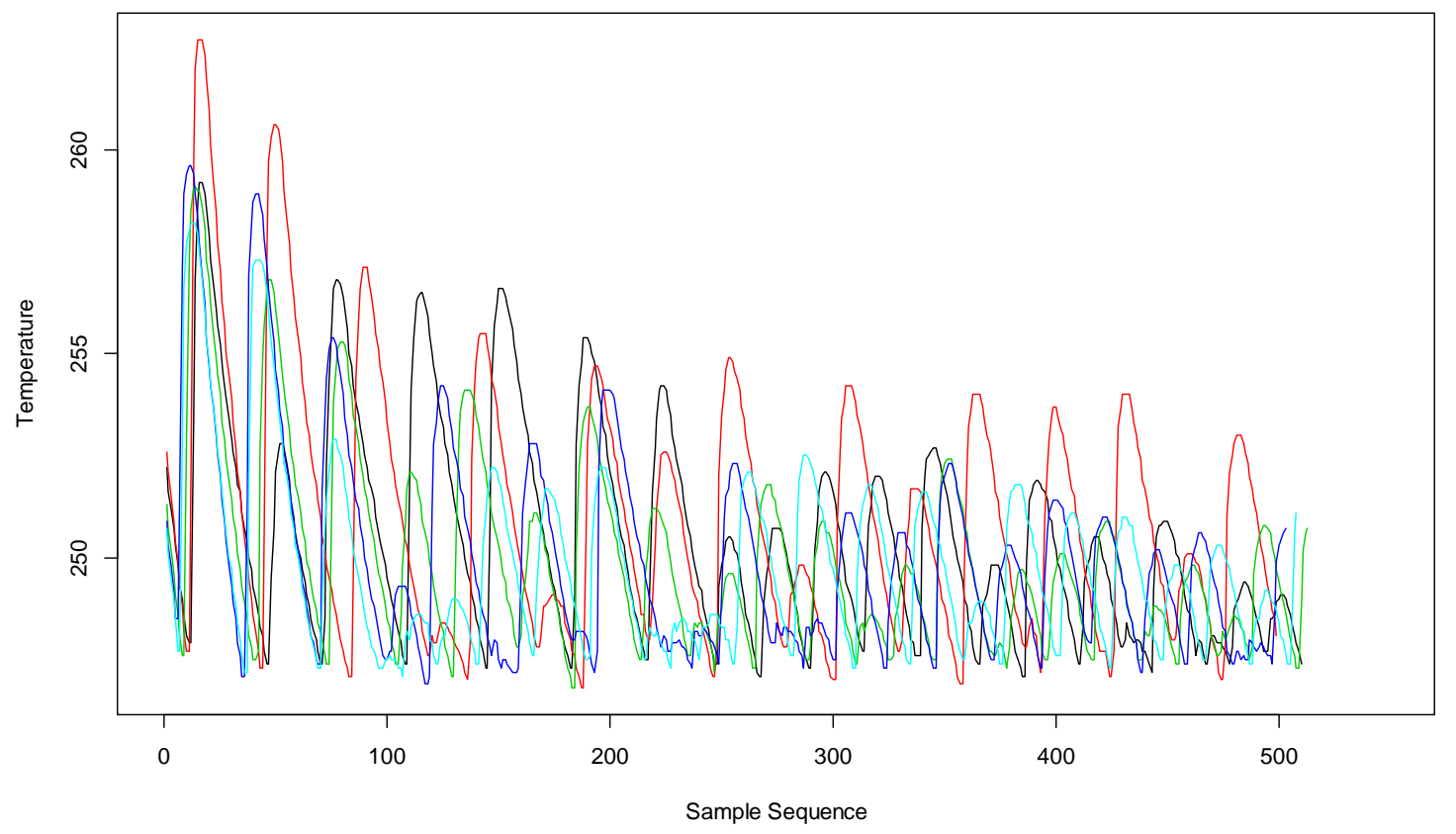

Figure 2. In-control wave profiles.

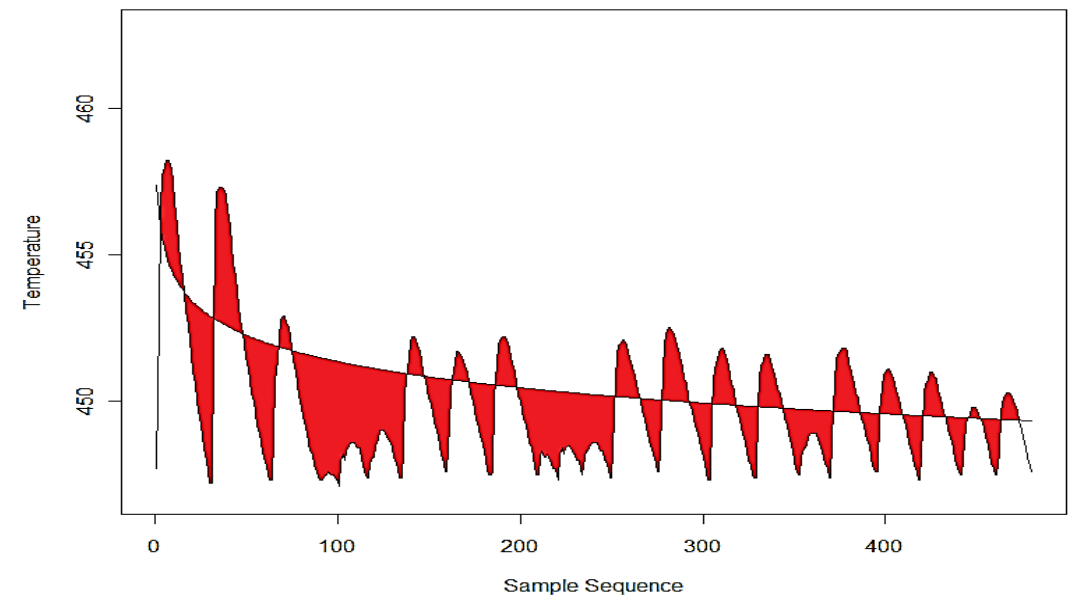

Figure 3. The enclosed area between the fitted exponential decay function and the profile. 


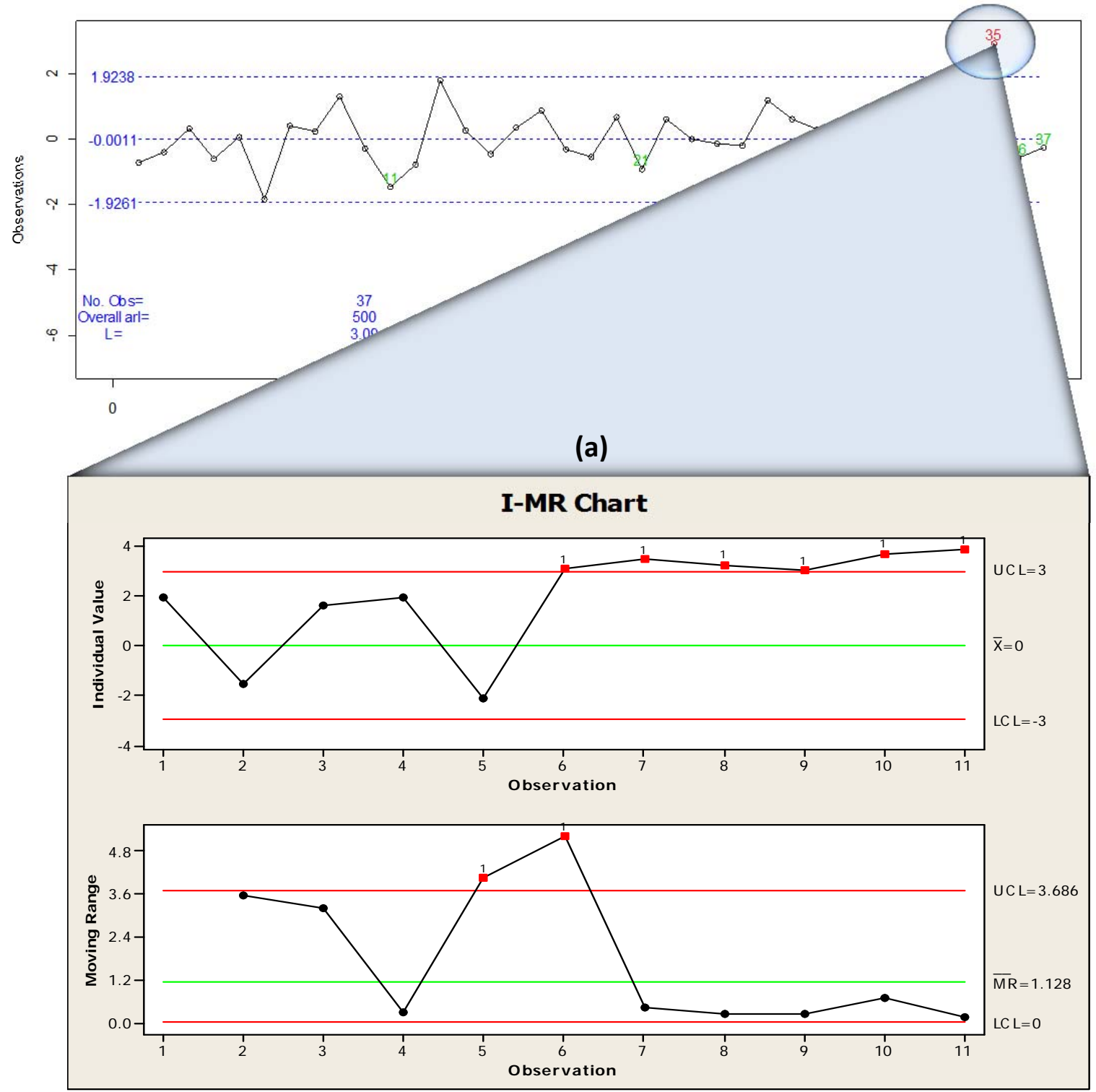

(b)

Figure 4. A comparison of (a) the original work: each point represents a summary of a profile, and (b) the proposed work: each point $\left(e_{i t}\right)$ is based on a fraction of an entire profile. 


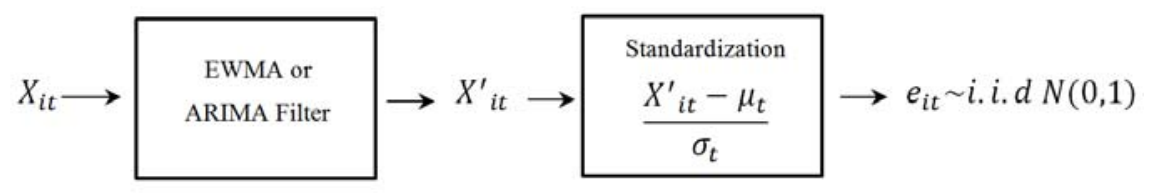

Figure 5. A scheme of the first proposed methodology.

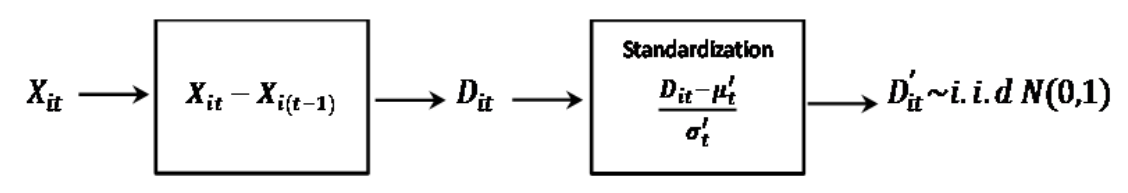

Figure 6. A scheme of the second proposed methodology.

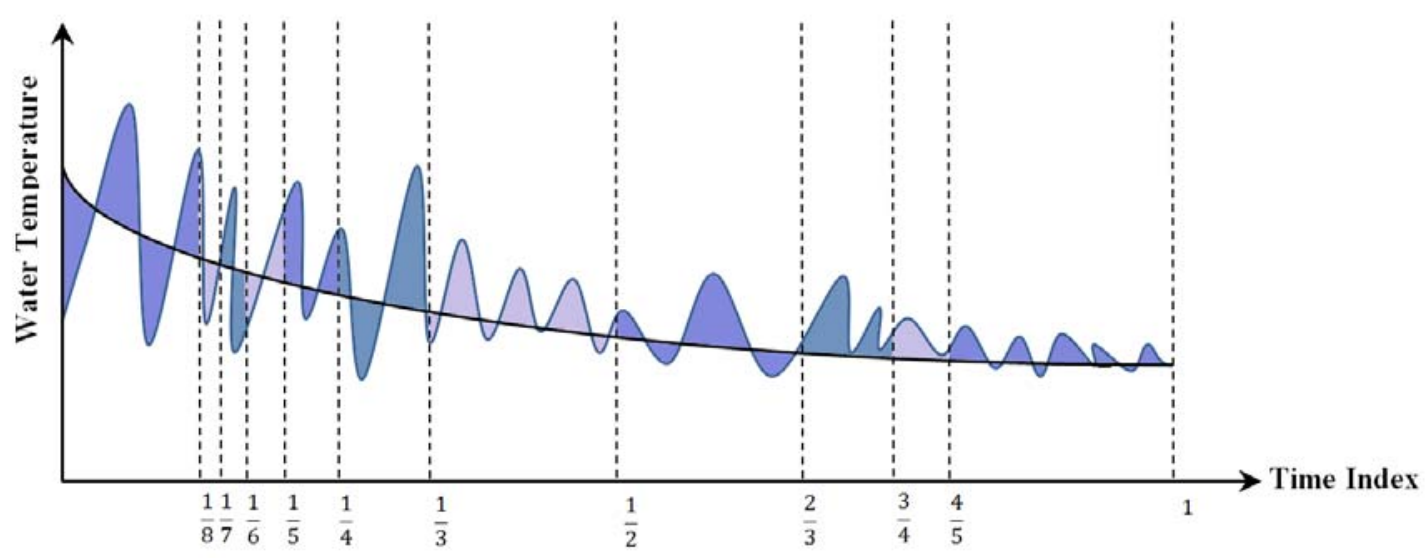

Figure 7. Detection Point at Time Index t 


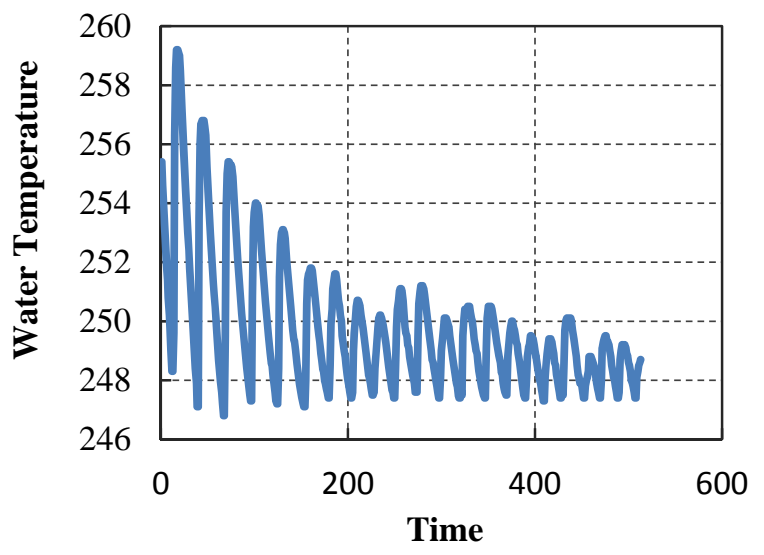

(a)

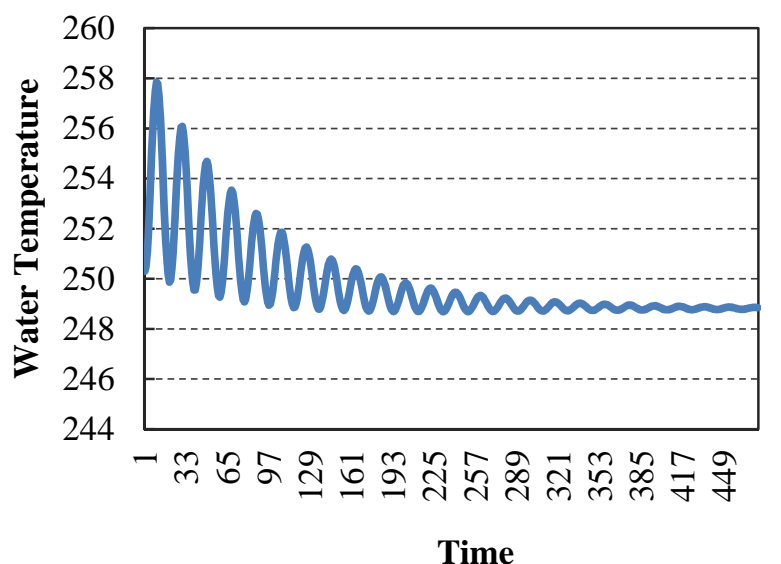

(b)

Figure 9. An Example of (a) a "typical" normal profile, and (b) a simulated normal profile.
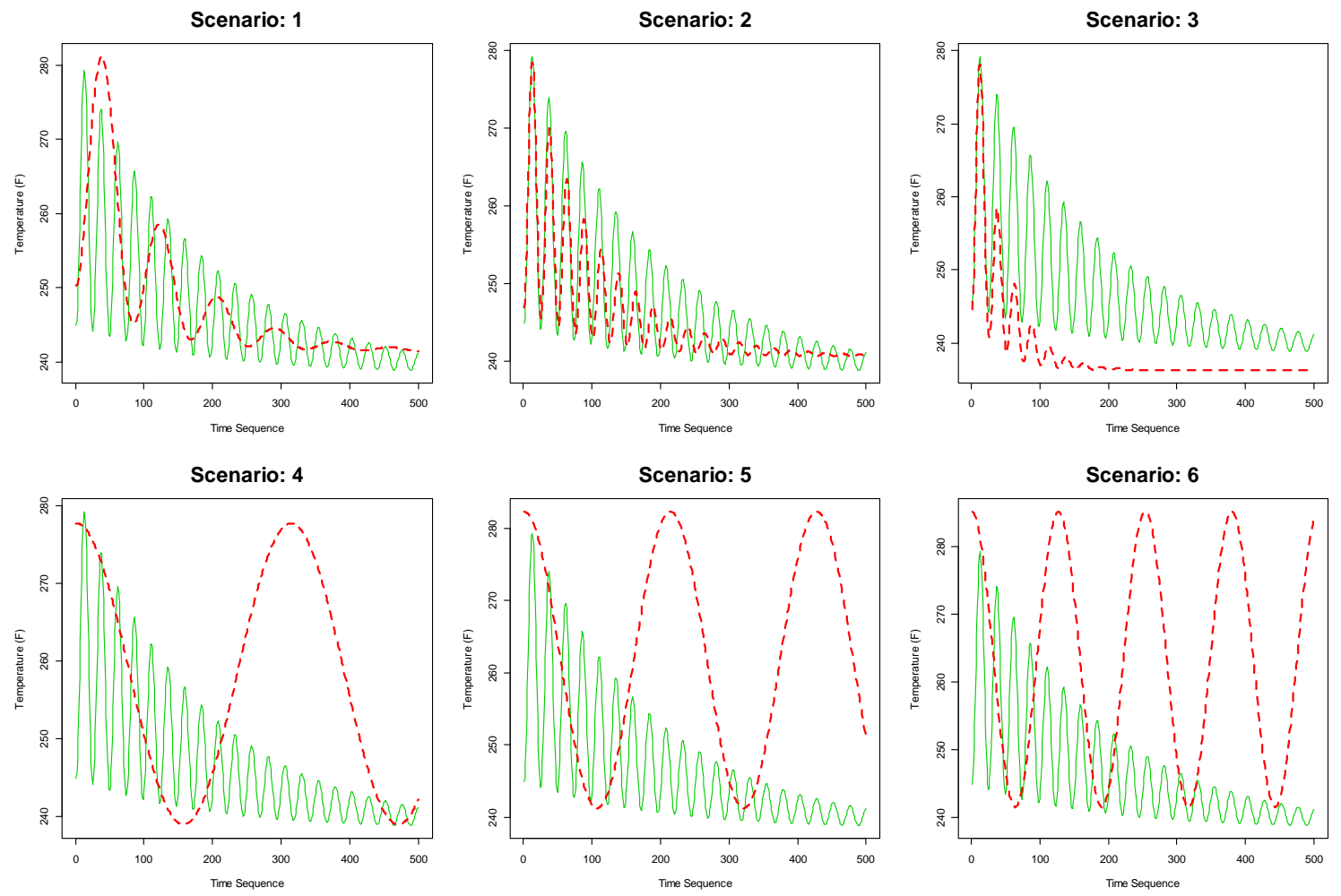

Figure 10. Six scenarios of abnormal profile patterns against a normal profile 


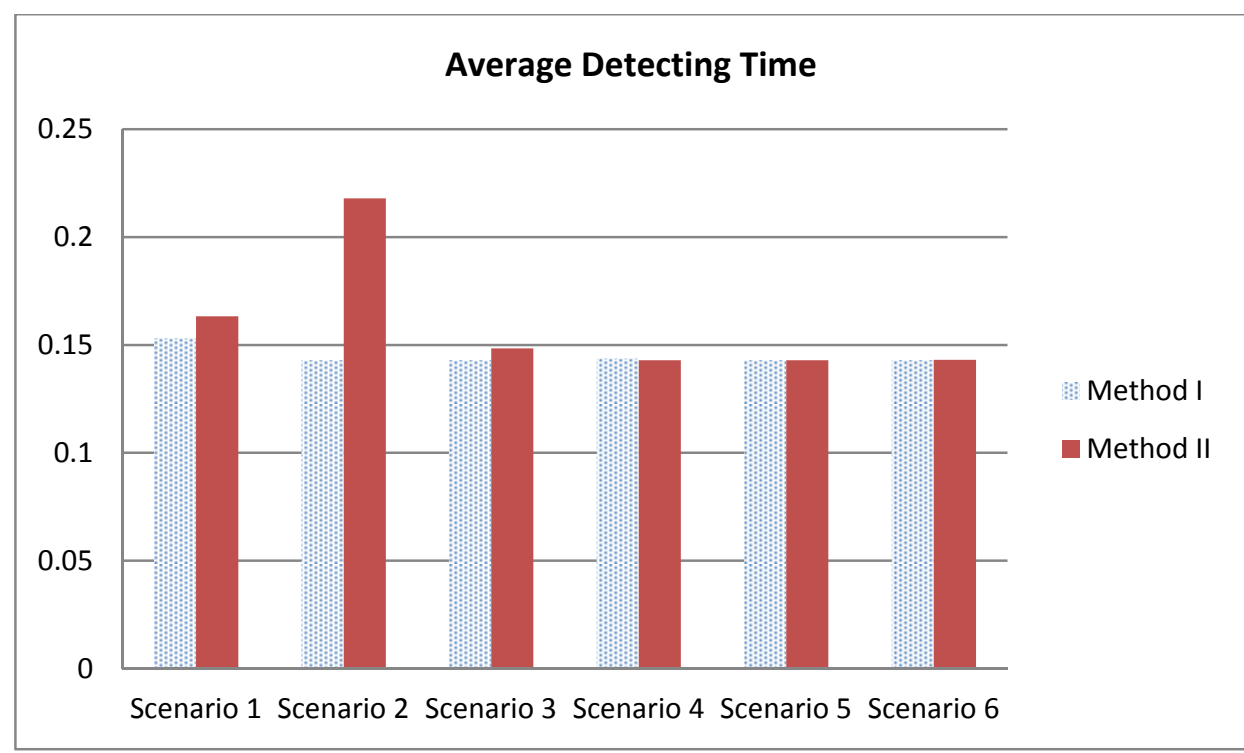

Figure 11. Average detecting time of method I and method II among all scenarios.

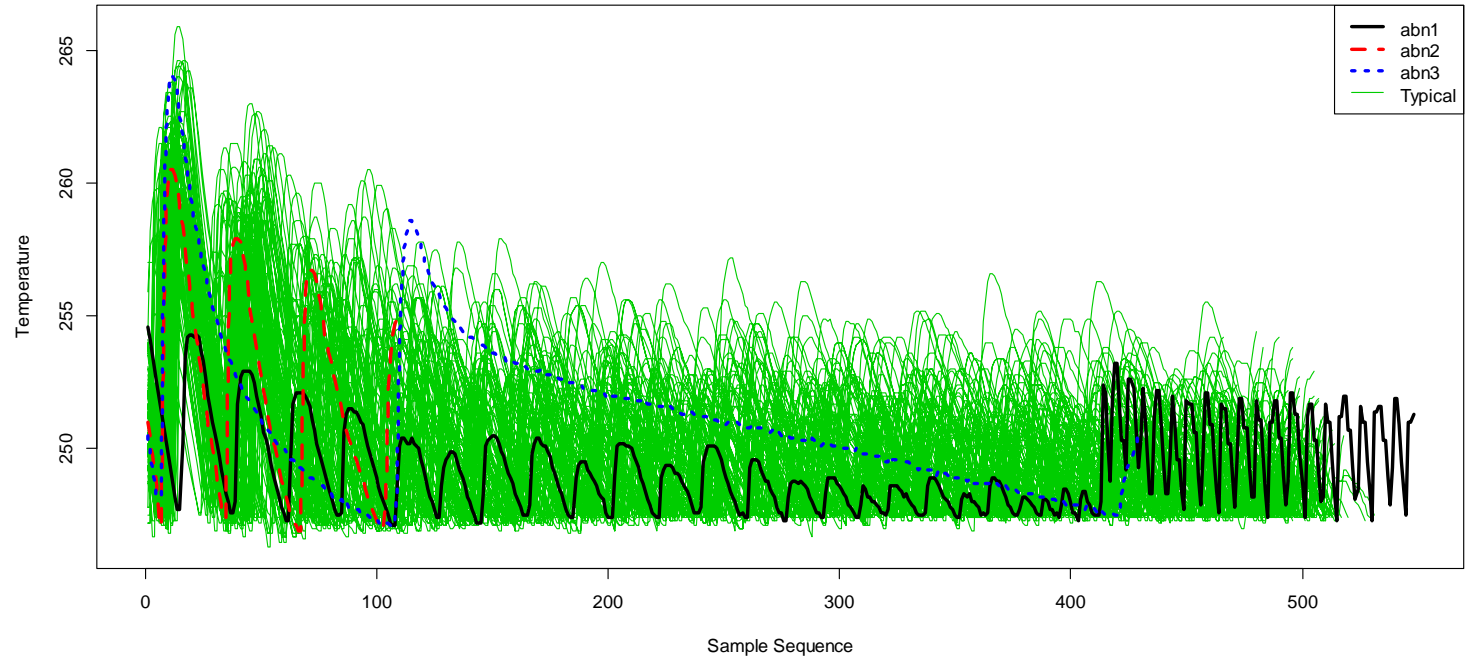

Figure 12. Three abnormal waveform profiles superimposed on 91 typical waveform profiles. 


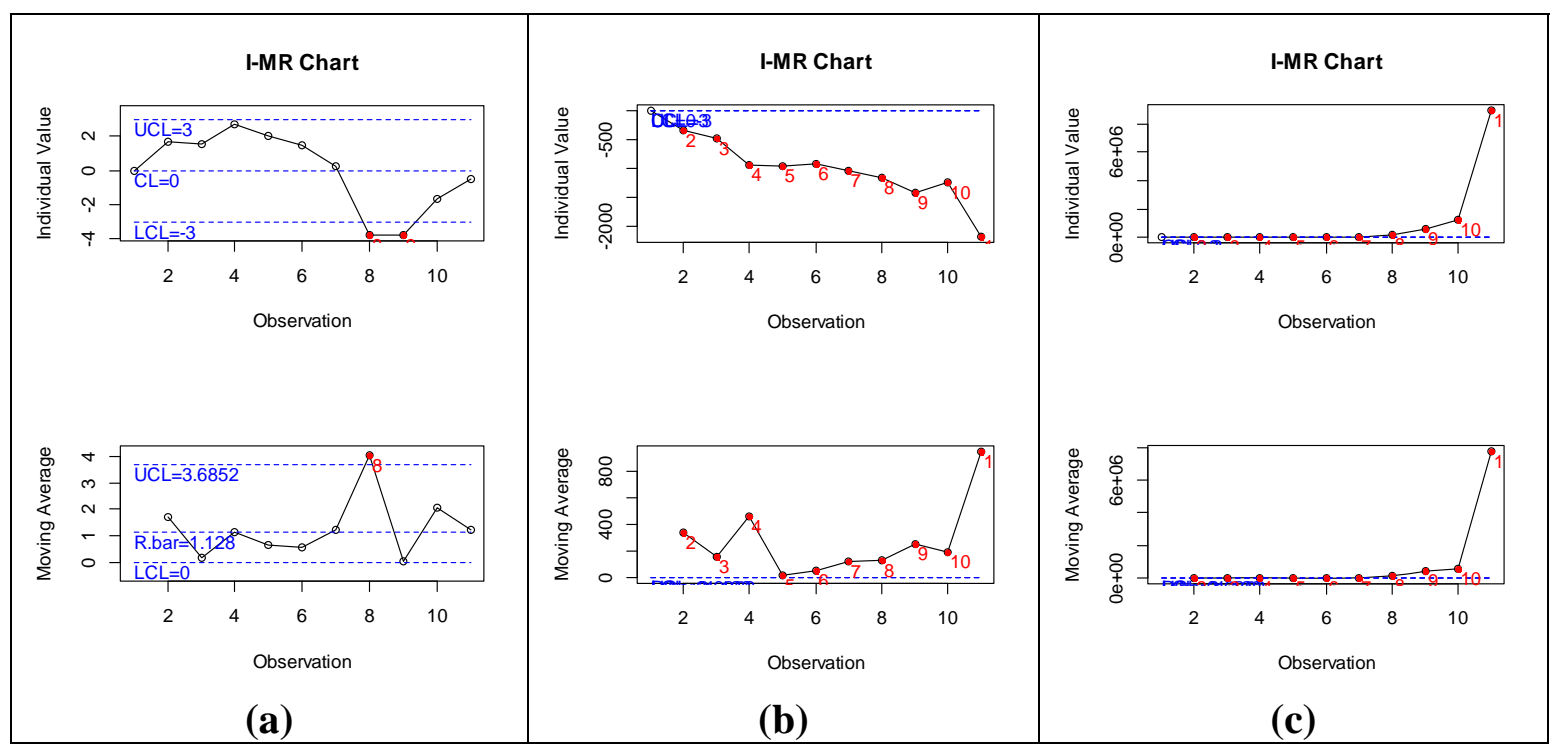

Figure 13. The results of method I on I-MR chart for (a) abn1; (b) abn2; (c) abn3.

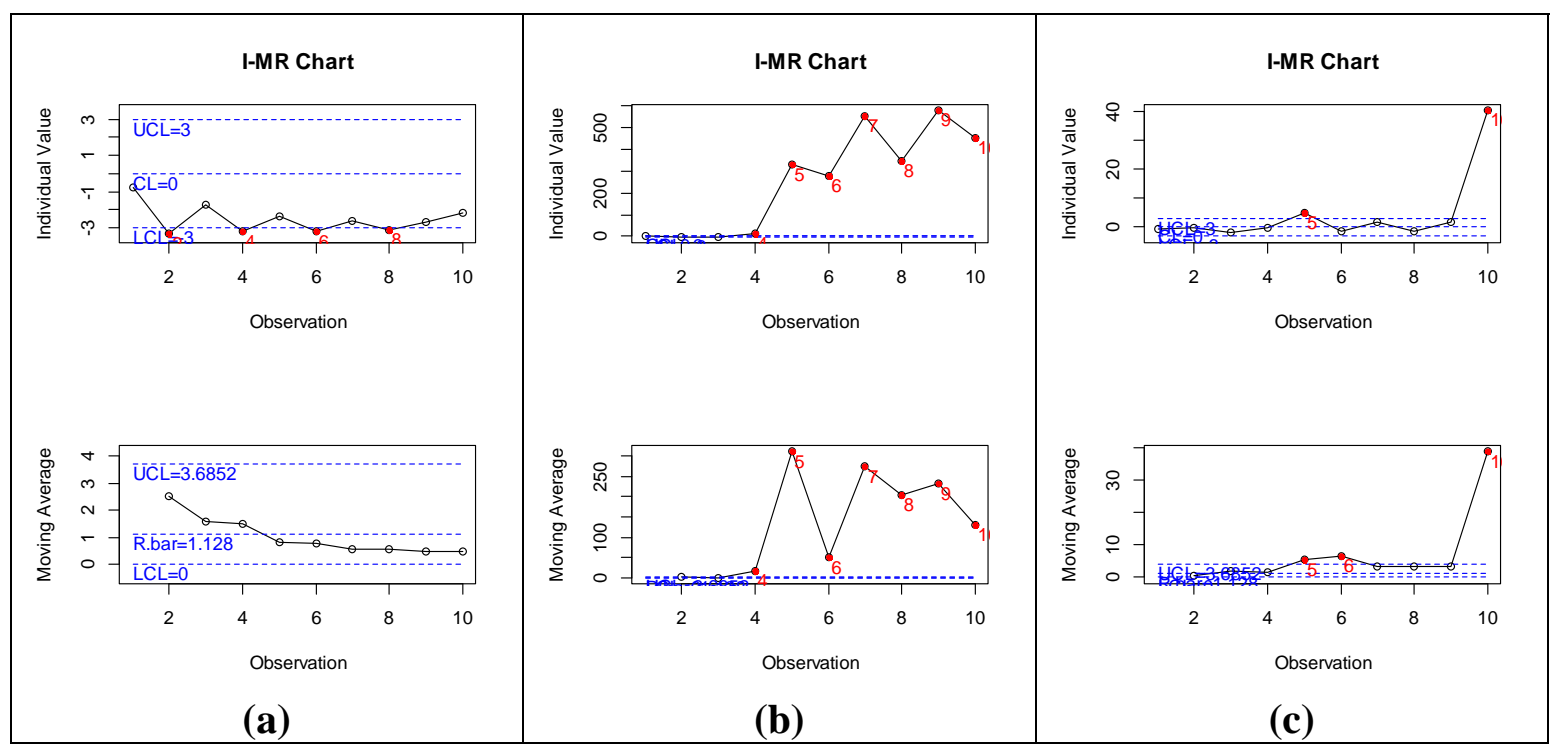

Figure 14. The results of method II on I-MR chart for (a) abn1; (b) abn2; (c) abn3. 


\section{Tables}

Table 1. Means and Standard Deviations of In-Control $D_{t}^{\prime}$ at Various $t$

\begin{tabular}{|c|c|c|c|c|c|c|c|c|c|c|c|}
\hline$i$ & 1 & 2 & 3 & 4 & 5 & 6 & 7 & 8 & 9 & 10 & 11 \\
\hline$t$ & $\frac{1}{8}$ & $\frac{1}{7}$ & $\frac{1}{6}$ & $\frac{1}{5}$ & $\frac{1}{4}$ & $\frac{1}{3}$ & $\frac{1}{2}$ & $\frac{2}{3}$ & $\frac{3}{4}$ & $\frac{4}{5}$ & 1 \\
\hline$\mu_{t}^{\prime}$ & 236.3 & 29.36 & 36.69 & 50.76 & 78.01 & 106.07 & 185.99 & 165.65 & 86.47 & 46.83 & 187.30 \\
\hline$\sigma_{t}^{\prime}$ & 74.53 & 16.68 & 20.19 & 24.67 & 44.60 & 49.80 & 96.83 & 76.82 & 52.55 & 32.71 & 97.96 \\
\hline
\end{tabular}

Table 2. Numerical Setting of Abnormal Profiles

\begin{tabular}{|c|l|c|}
\hline Scenario & Parameter & Equation \\
\hline 1 & $\begin{array}{l}m \sim N\left(1.5,0.005^{2}\right), n \sim N\left(0.2,0.005^{2}\right), \mathrm{A} \sim N\left(10,0.7^{2}\right), \\
f \sim N\left(260,2^{2}\right)\end{array}$ & (8) and (9) \\
\hline 2 & $\begin{array}{l}m \sim N\left(5,0.005^{2}\right), n \sim N\left(0.2,0.005^{2}\right), \mathrm{A} \sim N\left(10,0.7^{2}\right), f \sim N \\
\left(260,2^{2}\right)\end{array}$ & (8) and (9) \\
\hline 3 & $\begin{array}{l}m \sim N\left(5,0.005^{2}\right), n \sim N\left(0.5,0.005^{2}\right), \mathrm{A} \sim N\left(10,0.7^{2}\right), f \sim N \\
\left(260,2^{2}\right)\end{array}$ & (8) and (9) \\
\hline 4 & $m \sim N\left(0.4,0.005^{2}\right), \mathrm{A} \sim N\left(10,0.7^{2}\right), f \sim N\left(260,2^{2}\right)$ & (10) and (11) \\
\hline 5 & $m \sim N\left(0.6,0.005^{2}\right), \mathrm{A} \sim N\left(10,0.7^{2}\right), f \sim N\left(260,2^{2}\right)$ & (10) and (11) \\
\hline 6 & $m \sim N\left(1,0.005^{2}\right), \mathrm{A} \sim N\left(10,0.7^{2}\right), f \sim N\left(260,2^{2}\right)$ & (10) and (11) \\
\hline
\end{tabular}

Table 3. Simulation results of the proposed methods among all scenarios.

\begin{tabular}{|c|c|c|c|c|c|c|c|c|c|c|}
\hline Scenario & Method & TP & $\mathrm{TN}$ & FP & FN & Sensitivity $S$ & Specificity & y Accuracy & $\begin{array}{c}\text { Average } \\
\text { detecting } \\
\text { time }\end{array}$ & $\begin{array}{l}\text { Standard } \\
\text { deviation } \\
\text { of } \\
\text { detecting } \\
\text { time }\end{array}$ \\
\hline 1 & 1 & 0.98 & 1 & 0 & 0.02 & 0.98 & & 0.99 & 0.1530 & 0.0120 \\
\hline 1 & II & 0.99 & 1 & 0 & 0.01 & 0.99 & & 0.995 & 0.1633 & 0.0089 \\
\hline 2 & 1 & 0.98 & 1 & 0 & 0.02 & 0.98 & & 0.99 & 0.1429 & 0.0005 \\
\hline 2 & II & 0.99 & 1 & 0 & 0.01 & 0.99 & & 0.995 & 0.2179 & 0.0408 \\
\hline 3 & 1 & 0.98 & 1 & 0 & 0.02 & 0.98 & & 0.99 & 0.1429 & 0.0000 \\
\hline 3 & II & 0.99 & 1 & 0 & 0.01 & 0.99 & & 0.995 & 0.1484 & 0.0101 \\
\hline 4 & 1 & 0.98 & 1 & 0 & 0.02 & 0.98 & & 0.99 & 0.1437 & 0.0047 \\
\hline 4 & II & 0.99 & 1 & 0 & 0.01 & 0.99 & & 0.995 & 0.1429 & 0.0000 \\
\hline 5 & 1 & 0.98 & 1 & 0 & 0.02 & 0.98 & & 0.99 & 0.1429 & 0.0006 \\
\hline 5 & II & 0.99 & 1 & 0 & 0.01 & 0.99 & & 0.995 & 0.1429 & 0.0000 \\
\hline 6 & 1 & 0.98 & 1 & 0 & 0.02 & 0.98 & & 0.99 & 0.1429 & 0.0000 \\
\hline 6 & II & 0.99 & 1 & 0 & 0.01 & 0.99 & & 0.995 & 0.1431 & 0.0022 \\
\hline
\end{tabular}

